\title{
PROJETO DA MALHA DE CONTROLE DE UM PROCESSO DE HIDRÓLISE DE ANIDRIDO ACÉTICO EM CSTR
}

\author{
A. C. S. R. DIAS, B. R. BOTINHA, J. R. COSTA, C. S. O. TENIS e J. C. S. DUTRA \\ LAMCES - Laboratório de Métodos Computacionais, Controle e Estimação \\ Universidade Federal do Espírito Santo - Engenharia Química (campus de Alegre) \\ E-mail para contato: ana_csrd@hotmail.com; juliosdutra@yahoo.com.br
}

\begin{abstract}
RESUMO - Neste artigo, desenvolveu-se o projeto de uma estrutura de controle para um reator de hidrólise de anidrido acético por catálise ácida. A malha de controle projetada foi do tipo feedback, a qual empregou um controlador proporcional-integral-derivativo (PID). A variável manipulada utilizada foi a vazão de solução aquosa de ácido sulfúrico e a variável controlada foi a temperatura do reator. Os resultados obtidos demonstraram que o controlador foi capaz de manter a variável controlada em padrões desejáveis, sob diferentes condições operacionais.
\end{abstract}

\section{INTRODUÇÃO}

Sabe-se que o reator contínuo do tipo CSTR é utilizado para reações em fase líquida que requerem constante agitação. Devido a sua maior capacidade de produção, é mais utilizado em larga escala do que os reatores em batelada. Normalmente. para operações nesse tipo de reator, considera-se a mistura perfeita e, dessa forma, temperatura, composição e velocidade de reação serão uniformes em seu interior (Fogler, 2009; Levenspiel, 2000).

O trabalho de Jayakumar et al. (2011), utilizado como base para o presente estudo, fundamentou-se na análise de sensibilidade paramétrica e no estudo da dinâmica de um reator CSTR, encamisado de escala laboratorial, para hidrólise contínua de ácido acético catalisada por $\mathrm{H}_{2} \mathrm{SO}_{4}$. Essas ferramentas são de extrema importância e objetivam determinar a operação do processo em condições ideais. No entanto, os autores não consideraram o uso de estruturas de controle, que são cruciais para a implementação de políticas operacionais para manter o processo em torno da condição desejada (Seborg et al., 2011).

Com o presente trabalho, propõe-se um controlador capaz de manter a temperatura do reator em um valor desejado (set point), manipulando a vazão de solução aquosa de ácido sulfúrico. Para avaliar a eficiência do controlador, foram analisados os efeitos de degraus no set-point da temperatura e de perturbação na concentração inicial de anidrido acético.

\section{METODOLOGIA}

O modelo matemático do processo em análise considera como variáveis de entradas a temperatura de alimentação de anidrido acético, a vazão de solução aquosa de reagente e a concentração de alimentação de anidrido acético; e, como variáveis de estado e saída, a concentração de anidrido acético no reator, a temperatura de saída e a temperatura de liquido 
refrigerante. O modelo foi implementado no software SCILAB, sendo utilizada a rotina ode para realizar a integração das equações diferenciais. Inicialmente, para verificação da consistência deste presente trabalho, o resultado da simulação para a temperatura do reator foi comparado com aquele fornecido por Jayakumar et al. (2011).

Para análise da controlabilidade, o modelo foi linearizado numericamente em torno da condição de interesse $\left(5000 \mathrm{~mol} / \mathrm{m}^{3}\right.$ e $\left.40^{\circ} \mathrm{C}\right)$, possibilitando obter o modelo em espaço de estados (A, B, C e D) e, consequentemente, as funções de transferência do processo - G(s) (Seborg et al., 2011). Os polos e os zeros dessas funções, por sua vez, permitiram a análise da estabilidade, sendo utilizada especificamente a relação entre a vazão de reagente de solução aquosa (variável manipulada) com a temperatura do reator (variável controlada).

O projeto da malha de controle considerou um controlador proporcional-integralderivativo (PID) na forma paralela. Os parâmetros de sintonia do PID foram obtidos de modo a equilibrar o desempenho (tempo de resposta) e robustez (margens de estabilidade) (Seborg et al., 2011). Na sequência, foi obtida a função de transferência do sistema em malha fechada e, finalmente, foram realizados degraus no set-point da temperatura (teste servo) e na concentração inicial de anidrido acético (teste regulador). Com isso, analisou-se o desempenho do controlador para diferentes condições operacionais.

\section{RESULTADOS E DISCUSSÃO}

As Figuras 1(a) e 1(b) apresentam, respectivamente, o resultado fornecido pelo artigo de referência e o simulado neste trabalho. Pode-se observar a similaridade entre os gráficos, quando a capacitância da parede (Ws) é igual a $957 \mathrm{~J} /{ }^{\circ} \mathrm{C}$. Assim, é possível afirmar que a simulação realizada neste trabalho está de acordo com resultado fornecido pelo artigo. Deste modo, o modelo foi utilizado para o subsequente projeto do controlador.

Figura 1 - (a) Perfil de temperatura do reator fornecido por Jayakumar et al., 2010),

(b) Temperatura do reator simulada neste trabalho.

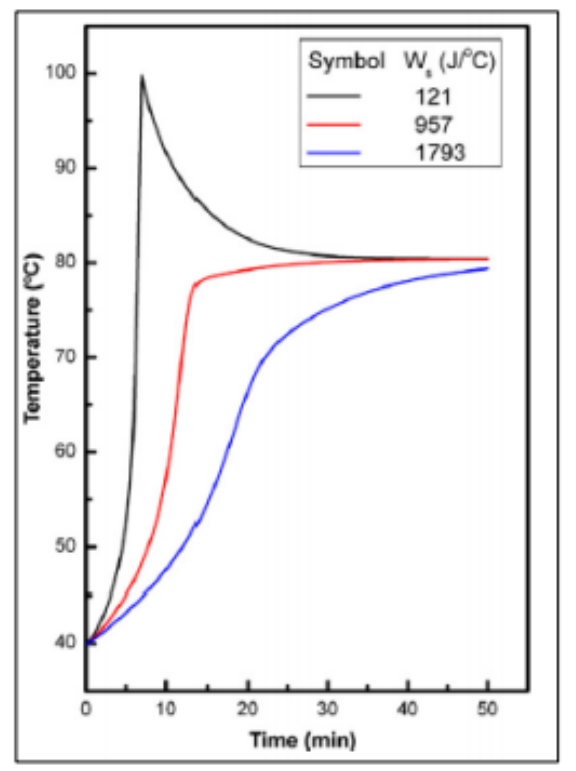

1 (a)

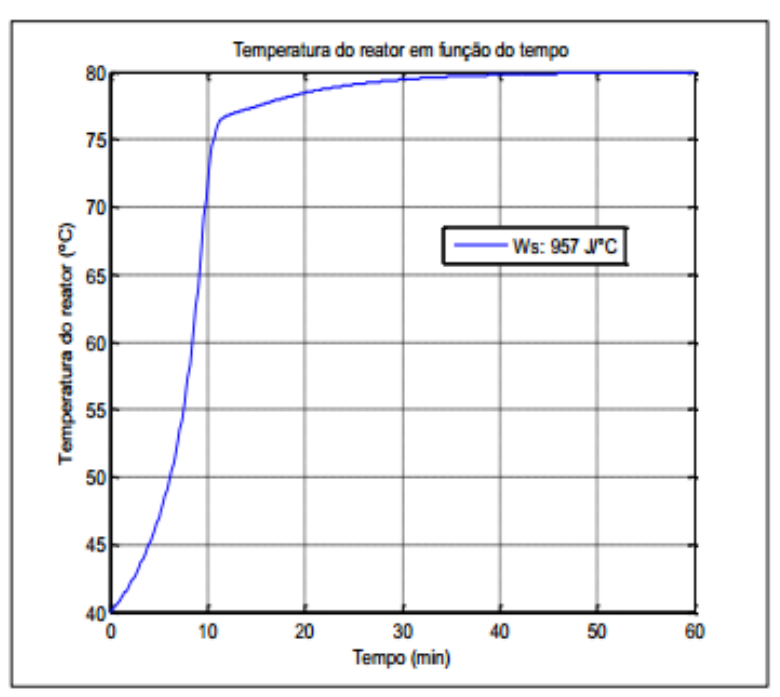

1(b) 
As matrizes de observabilidade $(\mathrm{O})$ e controlabilidade $(\mathrm{CO})$ do modelo linearizado e seus respectivos postos estão contidos nas Figuras 3 e 4 . Os postos dessas matrizes são iguais ao número de estados, de modo que o sistema pode ser considerado observável e controlável.

Figura 3 - Matriz de observabilidade.

$$
\mathrm{OB}=\left|\begin{array}{rrr}
1.0000 & 0 & 0 \\
0 & 1.0000 & 0 \\
0 & 0 & 1.0000 \\
-0.0033 & -0.6038 & 0 \\
0 & 0.0034 & 0.0011 \\
0 & 0.0046 & -0.0095 \\
0 & 0 & -0.0006 \\
0 & 0 & 0 \\
0 & 0 & 0.0001
\end{array}\right|
$$

Figura 4 - Matriz de controlabilidade.

$$
\mathrm{CO}=10^{4} \times\left|\begin{array}{rrrrrrrrr}
0 & 0 & 0 & 1.1012 & 0 & 0 & 0.0001 & 0 & 0 \\
-1.8238 & 0 & 0 & -0.0061 & 0 & 0 & 0 & 0 & 0 \\
0 & 0 & 0 & -0.0084 & 0 & 0 & 0.0001 & 0 & 0
\end{array}\right|
$$

Como o sistema é $3 \times 3$, foram obtidas nove funções de transferência do modelo linearizado, sendo representada pela Equação 1 a que relaciona a variável controlada com a variável manipulada (Gp). Como esta função não apresenta zeros positivos, a resposta inversa não é um comportamento esperado. Entretanto, há a presença de um polo positivo, o que evidencia a instabilidade do processo em malha aberta.

$$
G p(s)=\frac{-1,824 \times 10^{4} s^{2}-232,7 s-0,56894}{s^{3}+0,0094 s^{2}-1,046 \times 10^{-5} s-6,343 \times 10^{-8}}
$$

O projeto do controlador forneceu os parâmetros que se encontram na Tabela 1 .

Tabela 1 - Parâmetros do controlador PID.

\begin{tabular}{ccc}
\hline $\mathrm{K}_{\mathrm{c}}$ & $\tau_{\mathrm{I}}$ & $\tau_{\mathrm{D}}$ \\
\hline$-2,8607 \times 10^{-6}$ & $2,0559 \times 10^{3}$ & 67,9455
\end{tabular}

A Equação 2 apresenta a função de transferência do sistema em malha fechada $\left(\mathrm{G}_{\mathrm{MF}}\right)$. Os polos e zeros desta função apresentaram parte real negativa, demonstrando, assim, o comportamento estável do sistema em malha fechada para a sintonia empregada.

$$
\mathrm{G}_{\mathrm{MF}}(\mathrm{s})=\frac{0,403 \mathrm{~s}^{4}+0,0103 \mathrm{~s}^{3}+8,136 \times 10^{-5} \mathrm{~s}^{2}+1,942 \times 10^{-7} \mathrm{~s}+7,847 \times 10^{-11}}{60 \mathrm{~s}^{6}+7,507 \mathrm{~s}^{5}+0,567 \mathrm{~s}^{4}+0,011 \mathrm{~s}^{3}+7,988 \times 10^{-5} \mathrm{~s}^{2}+1,879 \times 10^{-7} \mathrm{~s}+7,847 \times 10^{-11}}(2)
$$


As Figuras 5 e 6 apresentam o comportamento da variável controlada e variável manipulada, respectivamente, quando o sistema é submetido a um degrau de magnitude $20{ }^{\circ} \mathrm{C}$ no set-point da temperatura do reator. Pode ser visto que o controlador foi capaz de levar a temperatura para o novo set-point. Não foram observadas oscilações nas respostas e o valor final da temperatura do reator foi obtido em um tempo de aproximadamente 5000 segundos.

Figura 5 - Resposta do sistema a um degrau de magnitude $20^{\circ} \mathrm{C}$ na temperatura do reator.

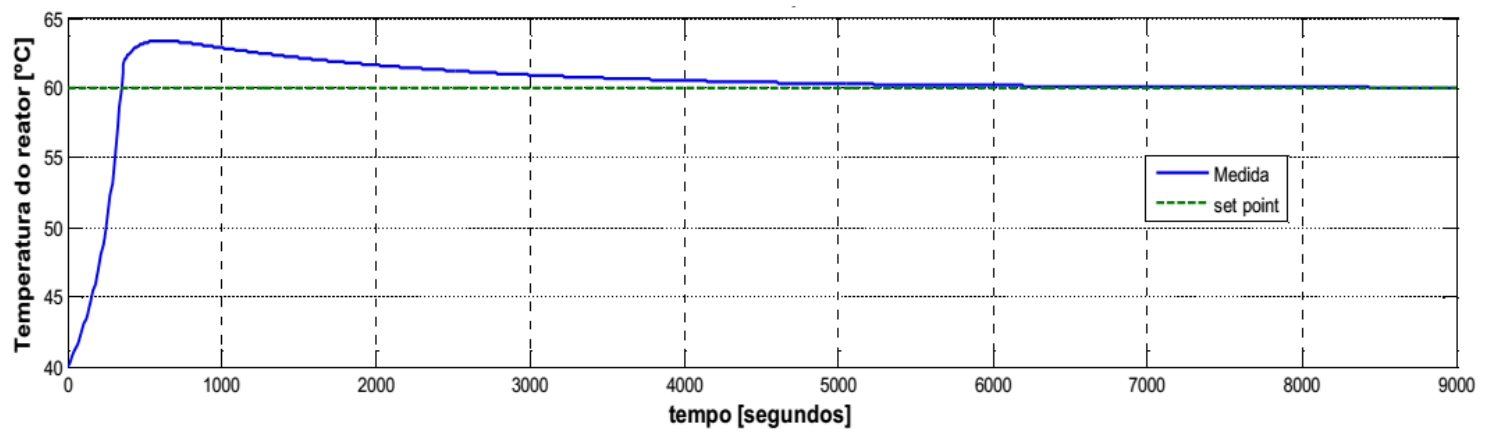

Figura 6 - Manipulação da vazão da solução aquosa de $\mathrm{H}_{2} \mathrm{SO}_{4}$.

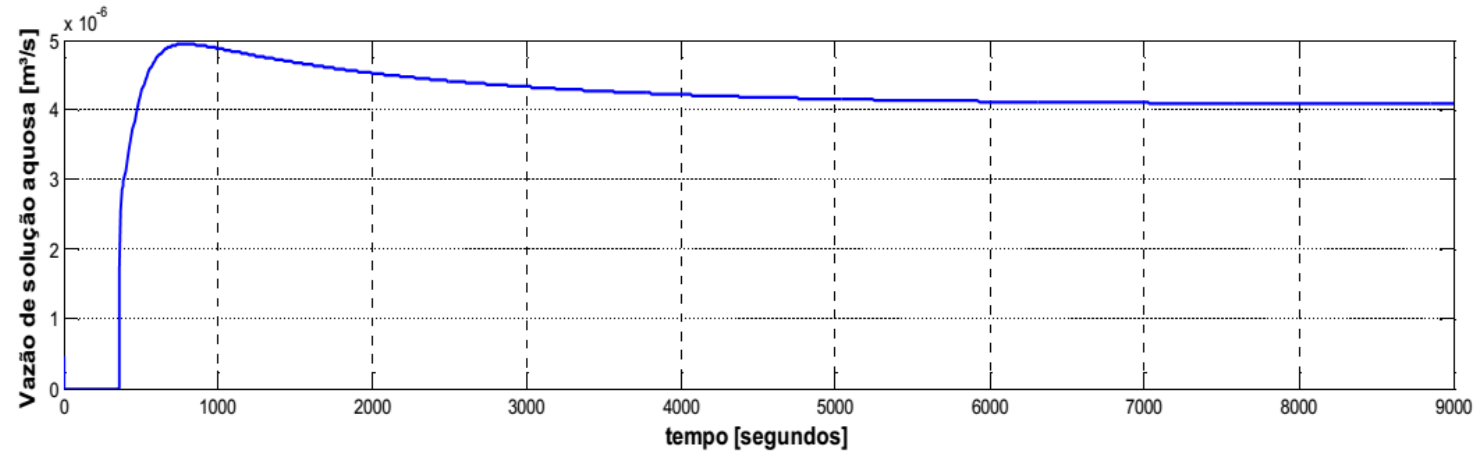

As Figuras 7 e 8 apresentam o comportamento da variável controlada e da variável manipulada quando o sistema é submetido a um degrau de magnitude $1000 \mathrm{~mol} / \mathrm{m}^{3}$ na concentração de alimentação de anidrido acético no tempo de 1000 segundos e a um degrau de magnitude $4{ }^{\circ} \mathrm{C}$ na temperatura de entrada dos reagentes no tempo de 3000 segundos. Durantes estes testes reguladores, o set-point da temperatura foi mantido em $40{ }^{\circ} \mathrm{C}$.

Figura 7 - Resposta do sistema aos testes reguladores, com set-point constante em $20^{\circ} \mathrm{C}$.

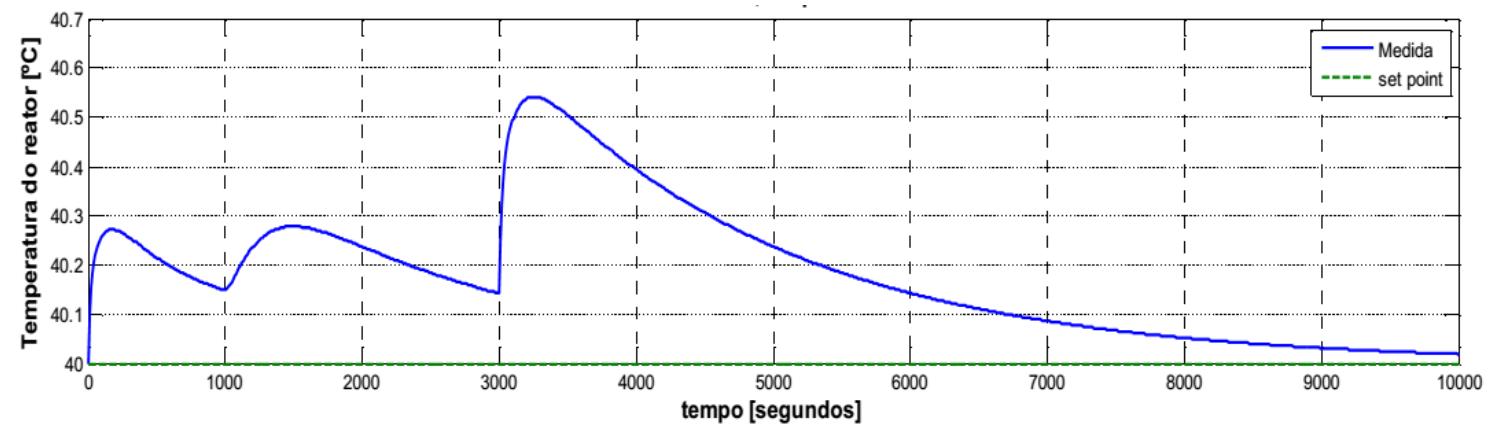


Figura 8 - Manipulação da vazão da solução aquosa de $\mathrm{H}_{2} \mathrm{SO}_{4}$ durante os testes reguladores.

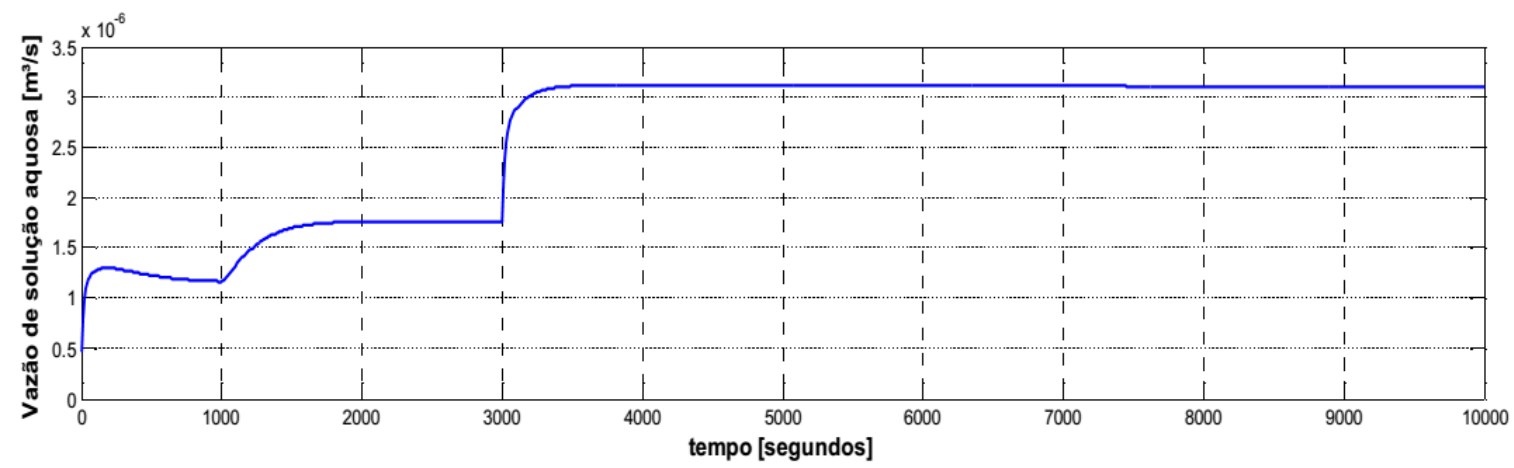

Foram realizados outros testes com diferentes valores para perturbações nas variáveis de entrada e no set-point e os resultados foram semelhantes. Contudo, para valores de set-point de temperatura superiores a $72{ }^{\circ} \mathrm{C}$, o controlador mostrou-se ineficiente, já que não foi possível manter a variável controlada no valor desejado e ocorreu offset considerável. Uma das possíveis causas para esse comportamento pode ser a existência de múltiplos estados estacionários na dinâmica do processo.

\section{CONCLUSÃO}

Foi possível implementar e simular o modelo fenomenológico do processo. Realizaramse as análises necessárias para obtenção de um controlador PID, visando manter a temperatura do reator em um valor desejado. Foram realizados testes com diferentes valores para perturbações nas variáveis de entrada e no set-point, sendo que o controlador projetado mostrou comportamento robusto e satisfatório. Para trabalhos futuros, sugere-se a implementação de um controlador de modelo interno (IMC). Espera-se que, com este método, sejam obtidos resultados melhores, pois o mesmo considera o efeito das perturbações sobre a dinâmica do processo.

\section{REFERÊNCIAS}

FOGLER, H. Scott. Elementos de engenharia das reações químicas. 4. ed. Rio de Janeiro: LTC, 2009.

JAYAKUMAR N.S. AGRAWAL A. HASHIM M.A. SAHU J.N. Experimental and theoretical investigation of parametric sensitivity and dynamics of a continuous stirred tank reactor for acid catalyzed hydrolysis of acetic anhydride. Computers \& Chemical Engineering, v. 35, PP. 1295-1303, 2011.

LEVENSPIEL, Octave. Engenharia das reações químicas. 3. ed. São Paulo: Editora Edgard Blucher, 2000

SEBorg, D. E., EDGAR, T. F., MELliCHAMP, D. A. Process Dynamics and Control, $3^{\text {rd }}$ ed. New York, USA: John Wiley and Sons, 2011. 